\title{
Orlistat, a novel potent antitumor agent for ovarian cancer: proteomic analysis of ovarian cancer cells treated with Orlistat
}

\author{
HUI-QIONG HUANG ${ }^{1 *}$, JING TANG $^{1 *}$, SHENG-TAO ZHOU ${ }^{1}$, TAO YI $^{1}$, HONG-LING PENG $^{1}$, GUO-BO SHEN ${ }^{2}$,

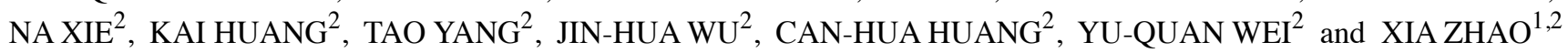 \\ ${ }^{1}$ Gynecological Oncology of Biotherapy Laboratory, Department of Gynecology and Obstetrics, \\ West China Second Hospital, Sichuan University, Chengdu, Sichuan; ${ }^{2}$ State Key Laboratory of Biotherapy \\ and Cancer Center, West China Hospital, Sichuan University, Chengdu, Sichuan, P.R. China
}

Received February 9, 2012; Accepted March 19, 2012

DOI: 10.3892/ijo.2012.1465

\begin{abstract}
Orlistat is an orally administered anti-obesity drug that has shown significant antitumor activity in a variety of tumor cells. To identify the proteins involved in its antitumor activity, we employed a proteomic approach to reveal protein expression changes in the human ovarian cancer cell line SKOV3, following Orlistat treatment. Protein expression profiles were analyzed by 2-dimensional polyacrylamide gel electrophoresis (2-DE) and protein identification was performed on a MALDI-Q-TOF MS/MS instrument. More than 110 differentially expressed proteins were visualized by 2-DE and Coomassie brilliant blue staining. Furthermore, 71 proteins differentially expressed proteins were positively identified via mass spectrometry (MS)/MS analysis. In particular, PKM1/2, a key enzyme involved in tumorigenesis, was found to be significantly downregulated in SKOV3 cells following treatment with Orlistat. Moreover, PKM1/2 was proved to be downregulated in SKOV3 cells by western blot analysis after treatment with Orlistat. Taken together, using proteomic tools, we identified several differentially expressed proteins that underwent Orlistat-induced apoptosis, particu-
\end{abstract}

Correspondence to: Professor Xia Zhao, Gynecological Oncology of Biotherapy Laboratory, Department of Gynecology and Obstetrics, West China Second Hospital, Sichuan University, No. 20, Section 3 South People's Road, Chengdu, Sichuan 610041, P.R. China

E-mail:xia-zhao@126.com

"Contributed equally

Abbreviations: 2-DE, two-dimensional polyacrylamide gel electrophoresis; MALDI-Q-TOF, matrix-assisted laser desorption ionization quadrupole time-of-flight; MOWSE, molecular weight search; ALODA, aldolase A; LDHA, L-lactate dehydrogenase A chain; KPYM, pyruvate kinase muscle isozyme; MS, mass spectrometry; MTT, 3-(4,5-dimethylthiazol-2-yl)-2,5-diphenyl tetrazolium bromide; PI, propidium iodide; CAPS, calcyphosine; FAS, fatty-acid synthase

Key words: Orlistat, antitumor agent, proteomics, ovarian cancer larly PKM2. These changes confirmed our hypothesis that Orlistat is a potential inhibitor of ovarian cancer and can be used as a novel adjuvant antitumor agent.

\section{Introduction}

In the 1920s, the Nobel Prize winner Otto Warburg observed a marked increase in glycolysis and enhanced lactate production in tumor cells even when maintained in conditions of high oxygen tension (termed Warburg effect), leading to widespread concerns about the metabolic changes in human types of cancer (1). Either as a consequence or as a cause, alterations of cancer cell-intrinsic metabolism have been considered as essential hallmarks of cancer. Among these metabolic changes, de novo fatty acid biosynthesis was found elevated in the majority of human types of cancer, such as prostate (2), colorectal (3), ovarian (4), bladder (5), esophageal (6), gastric (7), lung (8), endometrial (9), breast (10) and soft tissue sarcomas (11). Fatty acid synthase (FASN) is regarded as a key regulator of de novo fatty acid synthesis and was widely found upregulated in a wide variety of human malignancies and their pre-neoplastic lesions. Recent studies also reveal that FASN is associated with the stage of cancer and indicate a poor prognosis (12). Thus, FASN could be considered as a reliable predictor of recurrence and disease-free survival along with neo-plastic stage (13). In vivo treatment with inhibitors of FASN has been proven to lead to markedly decreased survival in human cancer xenografts (14) and silencing of the FASN gene by siRNA also inhibits cancer cell growth and ultimately induces cancer cell apoptosis (15). Therefore, agents that inhibit FASN and the de novo fatty-acid synthesis pathways could be considered as novel antitumor strategies.

Orlistat, an anti-obesity drug approved by the US Food and Drug Administration, which possesses extremely low oral bio-availability (16), exhibits anti-proliferative and antitumor properties against prostate and breast cancer cells due to its ability to block the lipogenic activity of FASN (16), by acting on the 2.3-A-resolution crystal structure of the thioesterase domain of FASN (17). Orlistat negatively influences FASN activity and has a significant effect on the antitumor activity by inducing remarkable diversification such as a complete G2-M phase loss, S-phase accumulation and the 
emerging sub-G1 (apoptotic) cell increase, and repression of the promoter activity of Her2/neu gene (18).

Ovarian cancer is the most common malignancy of the female reproductive tract and is the leading cause of death from gynecologic types of cancer; it is currently the fifth leading cause of female cancer-related mortality (19). Finding a novel therapeutic approach is essential since the 5-year survival rate of women with ovarian cancer is low, despite the fact that significant progress has been made in the therapy of this disease (20).

2-DE based proteomics has been shown to be a powerful tool in rapidly profiling differentially expressed proteins associated with a number of diseases (21-23). In our study, we aimed to investigate the differential expression in Orlistattreated SKOV3 cells using a 2DE-MS-based proteomics approach, in order to better understand the molecular mechanisms underlying Orlistat-induced tumor repression. In total, more than 110 differentially expressed proteins were found altered between Orlistat-treated and untreated SKOV3 cells, and subsequently 71 proteins were identified by MS analysis. Furthermore, we showed that PKM2 was significantly downregulated in Orlistat-treated SKOV3 cells, which confirmed the antitumor properties of Orlistat, indicating that Orlistat can be used as a novel adjuvant antitumor agent for ovarian cancer patients.

\section{Materials and methods}

Cell culture and treatment. The human epithelial serous cystadenocarcinoma cell line SKOV3 was obtained from the American Type Culture Collection (ATCC, Rockville, MD). Cells were grown in Dulbecco's-modified Eagle's medium (DMEM, Gibco, USA) containing $10 \%$ fetal calf serum (Hyclone, USA), penicillin $\left(10^{7} \mathrm{U} / \mathrm{l}\right)$ and streptomycin $(10 \mathrm{mg} / \mathrm{l})$ at $37^{\circ} \mathrm{C}$ in a humidified chamber containing $5 \% \mathrm{CO}_{2}$. Orlistat was dissolved in dimethyl sulphoxide (DMSO). When the cells reached $50-70 \%$ confluency, the medium was replaced by a fresh culture medium containing Orlistat. Control cells were cultured in a medium containing an equal amount of DMSO instead of Orlistat. For 2-DE analysis, SKOV3 cells were treated with $20 \mathrm{mM}$ Orlistat for 4 days and the media were changed every day. Cells were washed twice by centrifugation in phosphate buffered saline (PBS) and transferred to sterile plastic tubes for storage at $-80^{\circ} \mathrm{C}$ prior to use.

Cell proliferation assay. Cell growth and viability were assessed using an MTT cell proliferation kit (Roche Applied Science). The cells were seeded on 96-well microplates at $2.0 \times 10^{3} /$ well. At 48, 72 and $96 \mathrm{~h}$, the cells were treated with different concentrations of Orlistat and incubated at $37^{\circ} \mathrm{C}$ in $5 \% \mathrm{CO}_{2}$. The cells were subsequently incubated with $10 \mu \mathrm{l}$ of MTT for $4 \mathrm{~h}$, then the media were removed and $150 \mu \mathrm{l}$ DMSO were added. We put the plate in a shaker before reading absorbance at 490-nm using a microplate reader (3550-UV, Bio-Rad, USA), after $20 \mathrm{~min}$ of incubation. The procedure was repeated three times with similar results. The following formula was used to calculate the inhibition rate of SKOV3 cell proliferation: (1-experimental group OD value/negative control OD value) x $100 \%$. Media-only treated (untreated) cells were considered as the negative control group.
$2-D E$ and image analysis. Cells $\left(1.3 \times 10^{8}\right)$ were lysed in $1 \mathrm{ml}$ lysis buffer (7 M urea, $2 \mathrm{M}$ thiourea, 4\% CHAPS, 100 mM DTT, 0.2\% pH 3.0-10.0 ampholyte, Bio-Rad, USA) containing protease inhibitor cocktail 8340 (Sigma, St. Louis, MO, USA). Samples were then kept on ice and sonicated for six cycles of $10 \mathrm{sec}$, with each cycle consisting of $5 \mathrm{sec}$ sonication, followed by a $10 \mathrm{sec}$ break. After centrifugation at $14,000 \mathrm{rpm}$ for $1 \mathrm{~h}$ at $4^{\circ} \mathrm{C}$, the supernatant was collected and the protein concentrations determined using the DC Protein Assay Kit (Bio-Rad). Protein samples (3 mg) were applied to an immobilized pH gradient (IPG) strip $(17 \mathrm{~cm}$, pH 3.0-10.0 NL, Bio-Rad) using a passive rehydration method. After 12-16 h of rehydration, the strips were transferred to an isoelectric focusing (IEF) cell (Bio-Rad) and focused for a total of $60,000 \mathrm{Vh}$. The second dimension was performed using $12 \%$ equilibration. The gels were stained using CBB R-250 (Merck, Germany) and scanned with a Bio-Rad GS-800 scanner. Triplicate samples were analyzed at each time point of treatment to ensure the reproducibility of analyses. The maps were analyzed by PDQuest software Version 6.1 (Bio-Rad). Each gel spot was normalized as a percentage of the total quantity of all spots in that gel and evaluated in terms of OD. Only those spots that changed consistently and significantly (>2.0-fold) were selected for MS analysis.

In-gel digestion. In-gel digestion of proteins was carried out using MS-grade Trypsin Gold (Promega, Madison, WI, USA), according to the manufacturer's instructions. Briefly, spots were cut out of the gel (1-2 mm diameter) using a razor blade, and destained twice with $100 \mathrm{mM} \mathrm{NH}_{4} \mathrm{HCO}_{3} / 50 \%$ acetonitrile $(\mathrm{ACN})$ at $37^{\circ} \mathrm{C}$ for $45 \mathrm{~min}$ in each treatment. Following dehydration and drying, the gels were pre-incubated in 10-20 $\mu \mathrm{l}$ trypsin solution for $1 \mathrm{~h}$. Samples were then added in adequate digestion buffer $\left(40 \mathrm{mM} \mathrm{NH} \mathrm{HCO}_{3} / 10 \% \mathrm{ACN}\right)$ to cover the gels and incubated overnight at $37^{\circ} \mathrm{C}$. Tryptic digests were extracted using MiliQ water initially, followed by extraction twice with $50 \%$ ACN $/ 5 \%$ trifluoroacetic acid (TFA) for $1 \mathrm{~h}$ each time. The combined extracts were dried in a vacuum concentrator at room temperature. The samples were then subjected to MS analysis.

MALDI-Q-TOF analysis and protein identification. Mass spectra were acquired using a quadrupole time-of-flight (Q-TOF) mass spectrometer (Micromass, Manchester, UK) with a matrix-assisted laser desorption ionization (MALDI) source (Micromass). Tryptic digests were dissolved in $5 \mu \mathrm{l}$ of $70 \% \mathrm{ACN} / 0.1 \% \mathrm{TFA}$, and then $1 \mu \mathrm{l}$ of the digestion was mixed with $1 \mu \mathrm{l}$ saturated $\alpha$-cyano-4-hydroxy-cinnamic acid (CHCA) in 50\% ACN/0.5\% TFA and spotted onto a 96-well target plate. MS/MS was performed in a data-dependent mode in which the top ten most abundant ions for each MS scan were selected for MS/MS analysis. The MS/MS data were acquired and processed using the MassLynx software (Micromass) and MASCOT was used to search the database. Database searches were carried out using the following parameters: database, Swiss-Prot; taxonomy, Homo sapien; enzyme, trypsin; and allowance of one missed cleavage. Carbamidomethylation was selected as a fixed modification and oxidation of methionine was allowed to be variable. The peptide and fragment mass tolerance were at 1 and $0.2 \mathrm{Da}$, respectively. The data format 
selected was Micromass PKL and the instrument selected was MALDI-Q-TOF. Proteins with probability-based MOWSE scores exceeding their threshold $(\mathrm{P}<0.05)$ were considered to be positively identified.

Western blot analysis. Collected cells were lysed in RIPA buffer (50 mM Tris-base, $1.0 \mathrm{mM}$ EDTA, $150 \mathrm{mM} \mathrm{NaCl}$, $0.1 \%$ SDS, $1 \%$ Triton X-100, $1 \%$ sodium deoxycholate, $1 \mathrm{mM}$ PMSF) to extract all the proteins and quantified by the DC protein assay Kit (Bio-Rad). Samples were separated by $12 \%$ SDS-PAGE and transferred to polyvinylidene difluoride (PVDF) membranes (Amersham Biosciences). The membranes were blocked overnight with PBS containing $0.1 \%$ Tween 20 in $5 \%$ skimmed milk at $4^{\circ} \mathrm{C}$, and subsequently probed by the primary antibodies: rabbit anti-PKM2 (diluted 1:500, Abcam, UK). Blots were incubated with secondary antibody conjugated to horseradish peroxidase for $2 \mathrm{~h}$ at room temperature. Target proteins were detected by enhanced chemiluminescence reagents (Amersham Pharmacia Biotech, Piscataway, USA), and $\beta$-actin was used as an internal control.

Statistics. All quantitative data are recorded as the means \pm SD. Comparisons between two groups were performed by Student's t-test. Differences among multiple groups were assessed by one-way ANOVA analysis. Relevance analysis of ordinal data was performed by cross $\chi^{2}$ test. A statistically significant difference was defined as $\mathrm{p}<0.05$.

\section{Results}

Proliferation activity of Orlistat-treated SKOV3 cells. The proliferation activity of Orlistat-treated SKOV3 cells was examined using the MTT assays. MTT results showed that the proliferation activity was suppressed by Orlistat in both a dose- and duration-dependent manner, and the proliferation ratio was decreased to $60 \%$ of the control value $96 \mathrm{~h}$ after treatment with Orlistat when the drug concentration was $20 \mathrm{mM}$, as shown in Fig. 1.

Proteomic analysis of Orlistat-treated SKOV3 cell protein expression compared with the parental SKOV3 cells. To explore the molecular mechanisms underlying the Orlistatinduced antitumor activity of SKOV3 cells, 2-DE based proteomics was used to profile differentially expressed proteins in SKOV3 cells treated with or without Orlistat. Image analysis was performed using PDQuest 7.1 software. Representative 2-DE maps are shown in Fig. 2. Approximately 1000-1100 protein spots were detected by CBB R-250 staining in a single 2-DE gel. Each protein spot was normalized as a percentage of the total intensity of all spots in the gel. By comparing 2-DE patterns, differentially expressed proteins were defined as statistically meaningful $(\mathrm{p}<0.05)$ based on both of the following two criteria: i) intensity alterations of $>2.0$-fold (t-test, $\mathrm{p}<0.05$ ) and ii) observed in at least 3 individual experiments. According to these criteria, a total of 111 spots were selected and analyzed using MALDI-Q-TOF tandem mass spectrometry. A total of 71 proteins from the 111 spots were identified (Fig. 2). As different isoforms of a protein might have distinct functions, each isoform/spot was considered to be a single protein for analysis in our study.

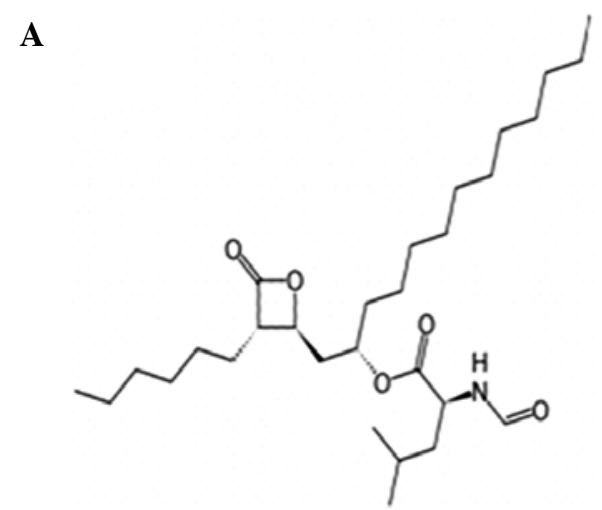

B

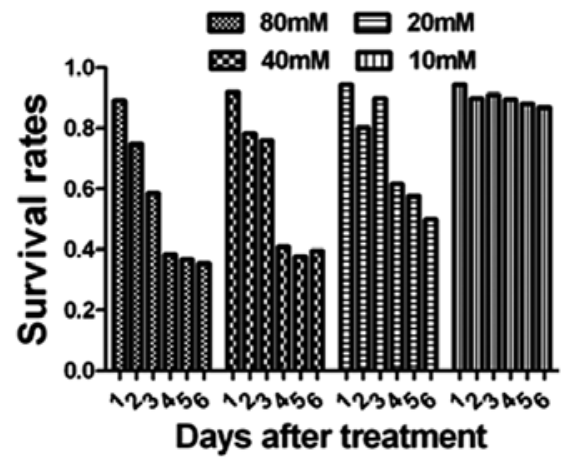

Figure 1. (A) Molecular structure of Orlistat. (B) Inhibitory effect of Orlistat on SKOV3 cell proliferation in vitro. Proliferation of the SKOV3 cells was assessed by the MTT assay. Data were assessed as percentage of cell viability in terms of media-only treated (non-treated) control cells at each drug concentration with different times and at each time with a different drug concentration. Orlistat caused a dose- and time-dependent inhibition of proliferation in vitro. Means $\pm \mathrm{SD}(\mathrm{n}=3)$. $\mathrm{P}<0.05$.

Protein identification and bioinformatics analysis. In total, 71 spots with differential expression levels were subjected to $\mathrm{MS} / \mathrm{MS}$ analysis. The MS/MS data were queried using the search algorithm MASCOT against the Expasy protein sequence database. Proteins were identified based on a number of criteria including PI, MW, the number of matched-peptides and MOWSE score (Table I and II).

The identified proteins were divided into various groups based on their biological functions and subcellular localization. This implicated roles in metabolism (32\%), protein folding (8\%), translation (5\%), protein modification (4\%), cell proliferation (15\%), apoptosis (10\%), signal transduction (14\%) and cell cytoskeleton (12\%). The proteins were found to be located in the cytoplasm (57\%), nucleus (11\%), mitochondrion (15\%), cell membrane (10\%) or were secreted (12\%) (Fig. 2). For a macroscopic presentation, cluster maps and protein interaction and function networks were generated using Cluster or the KEGG-based software tool Cytoscape, respectively. Twenty-three proteins, accounting for $32 \%$ of the proteins identified, were found to be associated with metabolism regulation. The metabolism-regulating proteins were grouped in different clusters. Pyruvate kinase isozymes M1/M2 were found to show one of the most significant differences in expression between SKOV3 cells treated with or without 


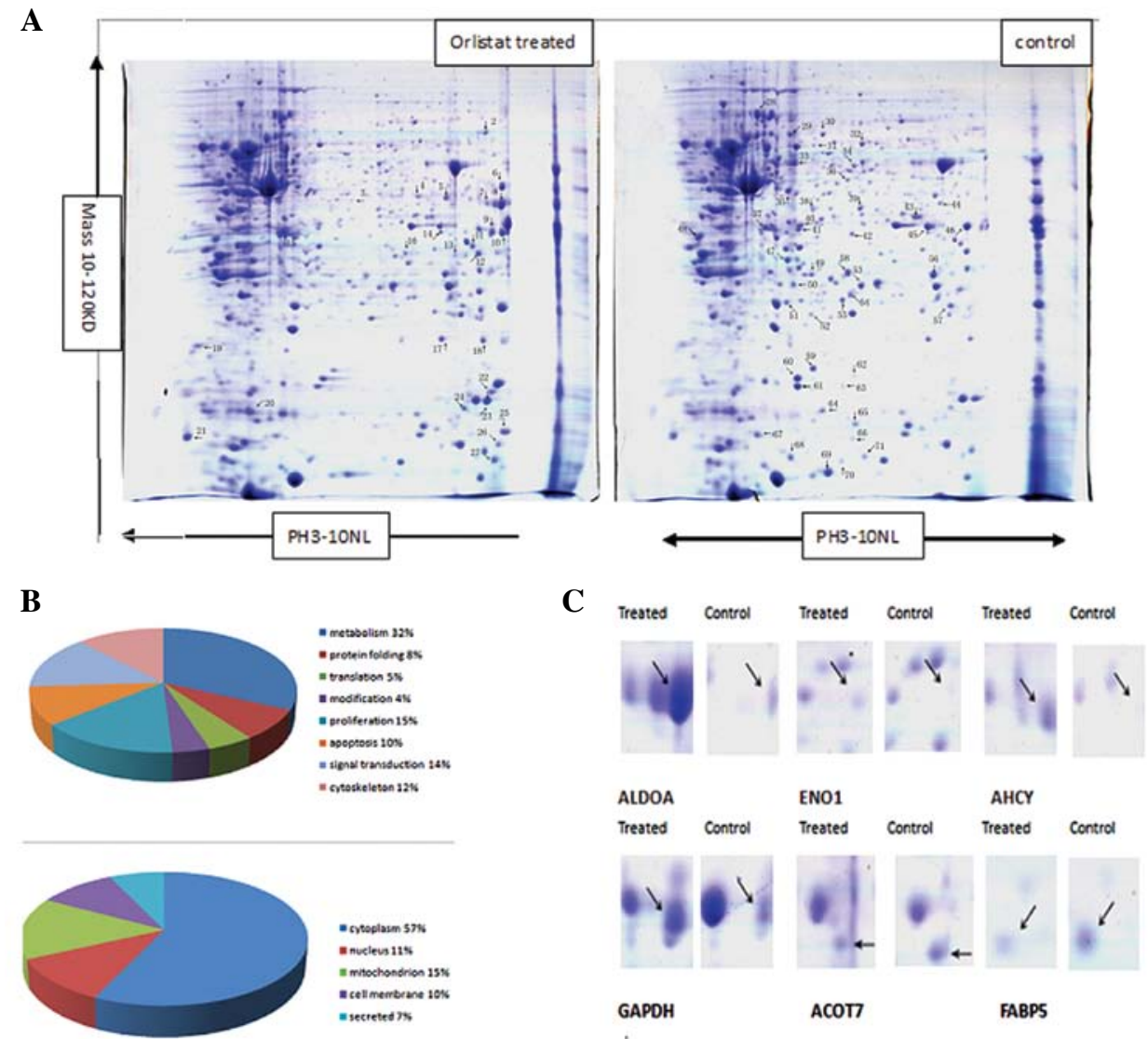

Figure 2. Comparison of the protein expression patterns between control and Orlistat-treated SKOV3 cells. (A) Representative 2-D gel images of the human ovarian cancer cell line SKOV3 treated with or without Orlistat. Total protein extracts were separated on $\mathrm{pH}$ 3.0-10.0 nonlinear IPG strips in the first dimension followed by $12 \%$ SDS-PAGE in the second dimension and visualized by Coomassie brilliant blue (CBB) staining. Seventy-one differentially expressed spots (27 upregulated and 44 downregulated in Orlistat-treated SKOV3 cells) were identified (as numbered). Details for each numbered spot are reported in Table I. (B) Seventy-one identified proteins were classified into 8 groups. These included metabolism (32\%), protein folding (8\%), translation (5\%), protein modification (4\%), cell proliferation (15\%), apoptosis (10\%), signal transduction (14\%) and cell cytoskeleton (12\%). These proteins were also found to be located in the cytoplasm (57\%), nucleus (11\%), mitochondrion (15\%), cell membrane (10\%) or were secreted (12\%). (C) Enlargement of selected regions. Spots selected are ALDOA (Spot 8), ENO1 (Spot 3), AHCY (Spot 7), GAPDH (Spot 9), ACOT7 (Spot 44), FABP5 (Spot 65).

Table I. Protein spots identified by MALDI-Q-TOF.

\begin{tabular}{|c|c|c|c|c|c|c|c|c|}
\hline $\begin{array}{l}\text { Spot } \\
\text { no. }\end{array}$ & $\begin{array}{c}\text { Accession } \\
\text { no. }^{\mathrm{b}}\end{array}$ & $\begin{array}{c}\text { Protein } \\
\text { name }^{a}\end{array}$ & $\begin{array}{l}\text { Gene } \\
\text { name }\end{array}$ & $\mathrm{Mw}^{\mathrm{c}}$ & $\mathrm{PI}^{\mathrm{c}}$ & $\begin{array}{l}\text { No. of } \\
\text { peptide }\end{array}$ & $\begin{array}{l}\text { Coverage } \\
(\%)\end{array}$ & Score \\
\hline \multicolumn{9}{|c|}{ Upregulated } \\
\hline 1 & P15311 & Ezrin & EZR & 69,484 & 5.94 & 5 & 6 & 133 \\
\hline 2 & P68104 & Elongation factor $1-\alpha 1$ & EEF1A1 & 50,451 & 9.10 & 15 & 28 & 179 \\
\hline 3 & P06733 & $\alpha$-enolas & ENO1 & 47,481 & 7.01 & 8 & 18 & 57 \\
\hline 4 & P04264 & Keratin, type II cytoskeletal 1 & KRT1 & 66,170 & 8.15 & 3 & 3 & 68 \\
\hline 5 & P50453 & Serpin B9 & SERPINB9 & 43,004 & 5.61 & 27 & 46 & 372 \\
\hline 6 & O00429 & Dynamin-1-like protein & DNM1L & 82,339 & 6.37 & 3 & 4 & 39 \\
\hline 7 & P23526 & $\begin{array}{l}\text { S-adenosyl-L-homocysteine } \\
\text { hydrolase }\end{array}$ & $\mathrm{AHCY}$ & 48,255 & 5.92 & 16 & 24 & 86 \\
\hline 8 & P04075 & Fructose-bisphosphate aldolase A & ALDOA & 39,851 & 8.30 & 56 & 68 & 723 \\
\hline 9 & P04406 & $\begin{array}{l}\text { Glyceraldehyde-3-phosphate } \\
\text { dehydrogenase }\end{array}$ & GAPDH & 36,201 & 8.57 & 27 & 55 & 400 \\
\hline 10 & P22626 & $60 \mathrm{~S}$ acidic ribosomal protein $\mathrm{P} 0$ & RPLP0 & 37,464 & 8.97 & 12 & 41 & 238 \\
\hline 11 & $\mathrm{P} 45880$ & $\begin{array}{l}\text { Voltage-dependent anion-selective } \\
\text { channel protein } 2\end{array}$ & VDAC2 & 32,060 & 7.49 & 10 & 29 & 91 \\
\hline 12 & P63244 & $\begin{array}{l}\text { Guanine nucleotide-binding } \\
\text { protein subunit } \beta \text {-2-like } 1\end{array}$ & GNB2L1 & 35,511 & 7.60 & 12 & 52 & 101 \\
\hline 13 & Q15056 & $\begin{array}{l}\text { Eukaryotic translation initiation } \\
\text { factor } 4 \mathrm{H}\end{array}$ & EIF4H & 27,425 & 6.67 & 7 & 28 & 125 \\
\hline
\end{tabular}


Table I. Continued.

\begin{tabular}{|c|c|c|c|c|c|c|c|c|}
\hline $\begin{array}{l}\text { Spot } \\
\text { no. }\end{array}$ & $\begin{array}{l}\text { Accession } \\
\text { no. }{ }^{\mathrm{b}}\end{array}$ & $\begin{array}{l}\text { Protein } \\
\text { name }^{\mathrm{a}}\end{array}$ & $\begin{array}{l}\text { Gene } \\
\text { name }\end{array}$ & $\mathrm{Mw}^{\mathrm{c}}$ & $\mathrm{PI}^{\mathrm{c}}$ & $\begin{array}{l}\text { No. of } \\
\text { peptide }\end{array}$ & $\begin{array}{l}\text { Coverage } \\
(\%)\end{array}$ & Score $^{\mathrm{d}}$ \\
\hline 14 & P04083 & Annexin A1 & Annexin I & 38,918 & 6.57 & 7 & 12 & 45 \\
\hline 15 & P52907 & F-actin-capping protein subunit $\alpha-1$ & CAPZA1 & 33,073 & 5.45 & 8 & 48 & 176 \\
\hline 16 & Q16740 & $\begin{array}{l}\text { Putative ATP-dependent } \\
\text { Clp protease proteolytic subunit, } \\
\text { mitochondrial }\end{array}$ & CLPP & 30,446 & 8.26 & 2 & 10 & 116 \\
\hline 17 & P07355 & Annexin A2 & ANXA2 & 38,808 & 7.57 & 45 & 54 & 885 \\
\hline 18 & Q99497 & Protein DJ-1 & PARK7 & 20,050 & 6.33 & 13 & 30 & 97 \\
\hline 19 & P30048 & $\begin{array}{l}\text { Thioredoxin-dependent peroxide } \\
\text { reductase, mitochondrial }\end{array}$ & PRDX3 & 28,017 & 7.67 & 9 & 20 & 115 \\
\hline 20 & P04792 & Heat shock protein $\beta$-1 & HSPB 1 & 22,826 & 5.98 & 6 & 37 & 48 \\
\hline 21 & P62158 & Calmodulin & CALM1 & 16,827 & 4.09 & 6 & 30 & 84 \\
\hline 22 & P60981 & Destrin & DSTN & 18,950 & 8.06 & 8 & 33 & 91 \\
\hline 23 & P00441 & Superoxide dismutase & SOD1 & 16,154 & 5.70 & 14 & 32 & 140 \\
\hline 24 & P30044 & $\begin{array}{l}\text { Peroxiredoxin-5, } \\
\text { mitochondrial }\end{array}$ & PRDX5 & 22,301 & 8.93 & 28 & 56 & 281 \\
\hline 25 & Q04837 & $\begin{array}{l}\text { Single-stranded DNA-binding } \\
\text { protein, mitochondrial }\end{array}$ & SSBP1 & 17,249 & 9.59 & 8 & 32 & 76 \\
\hline 26 & P07737 & Profilin-1 & PFN1 & 15,216 & 8.44 & 9 & 57 & 135 \\
\hline 27 & P61088 & $\begin{array}{l}\text { Ubiquitin-conjugating } \\
\text { enzyme E2 N }\end{array}$ & UBE2N & 17,184 & 6.13 & 7 & 36 & 42 \\
\hline \multicolumn{9}{|c|}{ Downregulated } \\
\hline 28 & P11142 & Heat shock cognate $71 \mathrm{kDa}$ protein & HSPA8 & 71,082 & 5.37 & 50 & 28 & 562 \\
\hline 29 & P35232 & Prohibitin & PHB & 29,843 & 5.57 & 29 & 51 & 440 \\
\hline 30 & P17987 & T-complex protein 1 subunit $\alpha$ & TCP1 & 60,819 & 5.80 & 28 & 37 & 170 \\
\hline 31 & P30101 & Protein disulfide-isomerase A3 & PDIA3 & 57,146 & 3.47 & 7 & 12 & 69 \\
\hline 32 & P78371 & T-complex protein 1 subunit $\beta$ & $\mathrm{CCT} 2$ & 57,794 & 6.01 & 36 & 49 & 446 \\
\hline 33 & P14618 & Pyruvate kinase isozymes M1/M2 & PKM2 & 58,470 & 7.96 & 26 & 36 & 309 \\
\hline 34 & Q9BWD1 & Acetyl-CoA acetyltransferase & ACAT2 & 41,838 & 6.47 & 12 & 41 & 86 \\
\hline 35 & P34949 & Mannose-6-phosphate isomerase & MPI & 47,196 & 5.62 & 2 & 7 & 38 \\
\hline 36 & P09972 & Fructose-bisphosphate aldolase C & ALDOC & 39,830 & 6.41 & 13 & 15 & 205 \\
\hline 37 & P68363 & Tubulin $\alpha-1 \mathrm{~B}$ & TUBA1B & 50,804 & 4.94 & 35 & 41 & 513 \\
\hline 38 & P50213 & $\begin{array}{l}\text { Isocitrate dehydrogenase (NAD) } \\
\text { subunit } \alpha, \text { mitochondrial }\end{array}$ & IDH3A & 40,022 & 6.47 & 22 & 49 & 152 \\
\hline 39 & P37837 & Transaldolase & TALDO1 & 37,688 & 6.36 & 23 & 37 & 363 \\
\hline 40 & P07195 & L-lactate dehydrogenase B chain & LDHB & 36,900 & 5.71 & 12 & 41 & 86 \\
\hline 41 & O00764 & Pyridoxal kinase & PDXK & 35,308 & 5.75 & 18 & 42 & 175 \\
\hline 42 & O00487 & $\begin{array}{l}26 \mathrm{~S} \text { proteasome non-ATPase } \\
\text { regulatory subunit } 14\end{array}$ & PSMD14 & 34,726 & 6.06 & 14 & 62 & 62 \\
\hline 43 & P11177 & $\begin{array}{l}\text { Pyruvate dehydrogenase E1 } \\
\text { component subunit } \beta \text {, } \\
\text { mitochondrial }\end{array}$ & PDHB & 39,550 & 6.20 & 14 & 16 & 186 \\
\hline 44 & O00154 & $\begin{array}{l}\text { Cytosolic acyl coenzyme A } \\
\text { thioester hydrolase }\end{array}$ & ACOT7 & 42,454 & 8.85 & 13 & 25 & 177 \\
\hline 45 & $\mathrm{P} 15121$ & Aldose reductase & AKR1B1 & 36,230 & 6.51 & 40 & 11 & 62 \\
\hline 46 & P00338 & L-lactate dehydrogenase A chain & LDHA & 36,950 & 8.44 & 40 & 52 & 319 \\
\hline 47 & P31937 & $\begin{array}{l}\text { 3-hydroxyisobutyrate } \\
\text { dehydrogenase, mitochondrial }\end{array}$ & HIBADH & 35,705 & 8.38 & 1 & 4 & 39 \\
\hline 48 & P12004 & Proliferating cell nuclear antigen & PCNA & 29,092 & 4.57 & 45 & 54 & 559 \\
\hline 49 & Q06830 & Peroxiredoxin-1 & PRDX1 & 22,324 & 8.27 & 2 & 18 & 60 \\
\hline 50 & Q13162 & Peroxiredoxin-4 & PRDX4 & 30,749 & 5.86 & 3 & 14 & 29 \\
\hline 51 & P00491 & Purine nucleoside phosphorylase & PNP & 32,325 & 6.45 & 4 & 5 & 35 \\
\hline 52 & P30086 & Phosphatidylethanolamine-binding & PEBP1 & 21,158 & 7.01 & 36 & 62 & 432 \\
\hline
\end{tabular}


Table I. Continued.

\begin{tabular}{|c|c|c|c|c|c|c|c|c|}
\hline $\begin{array}{l}\text { Spot } \\
\text { no. }\end{array}$ & $\begin{array}{c}\text { Accession } \\
\text { no. }\end{array}$ & $\begin{array}{c}\text { Protein } \\
\text { name }^{\mathrm{a}}\end{array}$ & $\begin{array}{l}\text { Gene } \\
\text { name }\end{array}$ & $\mathrm{Mw}^{\mathrm{c}}$ & $\mathrm{PI}^{\mathrm{c}}$ & $\begin{array}{l}\text { No. of } \\
\text { peptide }\end{array}$ & $\begin{array}{l}\text { Coverage } \\
(\%)\end{array}$ & Score $^{\mathrm{d}}$ \\
\hline 53 & P60174 & Triosephosphate isomerase & TPI1 & 26,938 & 6.45 & 15 & 57 & 136 \\
\hline 54 & P49720 & Proteasome subunit $\beta$ type- 3 & PSMB3 & 23,219 & 6.14 & 7 & 30 & 50 \\
\hline 55 & Q15185 & Prostaglandin E synthase 3 & PTGES3 & 18,971 & 4.35 & 6 & 18 & 90 \\
\hline 56 & Q99714 & $\begin{array}{l}\text { 3-hydroxyacyl-CoA dehydrogenase } \\
\text { type- } 2\end{array}$ & HSD17B10 & 27,134 & 7.66 & 42 & 73 & 632 \\
\hline 57 & P62826 & GTP-binding nuclear protein Ran & RAN & 24,579 & 7.01 & 4 & 18 & 19 \\
\hline 58 & P42126 & $\begin{array}{l}\text { Enoyl-CoA } \delta \text { isomerase } 1 \text {, } \\
\text { mitochondrial }\end{array}$ & ECI1 & 33,080 & 8.80 & 5 & 8 & 60 \\
\hline 59 & P15531 & Nucleoside diphosphate kinase A & NME1 & 17,309 & 5.83 & 19 & 59 & 192 \\
\hline 60 & P62937 & $\begin{array}{l}\text { Peptidyl-prolyl cis-trans } \\
\text { isomerase A }\end{array}$ & PPIA & 18,229 & 7.68 & 22 & 61 & 327 \\
\hline 61 & P16949 & Stathmin & STMN1 & 17,292 & 5.76 & 18 & 24 & 608 \\
\hline 62 & P24666 & $\begin{array}{l}\text { Low molecular weight } \\
\text { phosphotyrosine protein } \\
\text { phosphatase }\end{array}$ & $\mathrm{ACP} 1$ & 18,487 & 6.30 & 4 & 22 & 27 \\
\hline 63 & Q9UHV9 & Prefoldin subunit 2 & PFDN2 & 16,695 & 6.20 & 1 & 9 & 46 \\
\hline 64 & P62942 & Peptidyl-prolyl cis-trans isomerase & FKBP1A & 12,000 & 7.88 & 3 & 27 & 26 \\
\hline 65 & Q01469 & $\begin{array}{l}\text { Fatty acid-binding protein, } \\
\text { epidermal }\end{array}$ & FABP5 & 15,497 & 6.60 & 9 & 37 & 90 \\
\hline 66 & O60925 & Prefoldin subunit 1 & PFDN1 & 14,202 & 6.32 & 2 & 9 & 46 \\
\hline 67 & P35080 & Profilin-2 & PFN2 & 15,378 & 6.55 & 18 & 30 & 179 \\
\hline 68 & Q99584 & Protein S100-A13 & S100A13 & 11,464 & 5.91 & 2 & 22 & 28 \\
\hline 69 & P04264 & Keratin, type II cytoskeletal 1 & KRT1 & 66,170 & 8.15 & 8 & 11 & 28 \\
\hline 70 & P31949 & Protein S100-A11 & S100A11 & 11,847 & 6.56 & 4 & 15 & 36 \\
\hline 71 & P14174 & $\begin{array}{l}\text { Macrophage migration inhibitory } \\
\text { factor }\end{array}$ & MIF & 12,639 & 7.74 & 16 & 17 & 366 \\
\hline
\end{tabular}

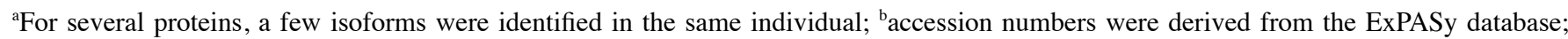
ctheoretical molecular mass (kDa) and PI from the ExPASy database; ${ }^{d}$ probability-based MOWSE (molecular weight search) scores.

Table II. Proteins identified to be involved in the metabolic process.

\begin{tabular}{|c|c|c|c|c|c|}
\hline $\begin{array}{l}\text { Spot } \\
\text { no. }\end{array}$ & $\begin{array}{l}\text { Accession } \\
\text { no. }\end{array}$ & $\begin{array}{l}\text { Protein } \\
\text { name }\end{array}$ & $\begin{array}{l}\text { Average } \\
\text { ratio }\end{array}$ & $\begin{array}{l}\text { Subcellular } \\
\text { location }\end{array}$ & $\begin{array}{l}\text { Main } \\
\text { function }\end{array}$ \\
\hline 3 & P06733 & $\alpha$-enolas & 10.54 & $\begin{array}{l}\text { Cell } \\
\text { membrane }\end{array}$ & Glycolysis \\
\hline 7 & P23526 & $\begin{array}{l}\text { S-adenosyl-L-homocysteine } \\
\text { hydrolase }\end{array}$ & 8.91 & Cytoplasm & $\begin{array}{l}\text { Control of } \\
\text { methylations }\end{array}$ \\
\hline 8 & P04075 & $\begin{array}{l}\text { Fructose-bisphosphate } \\
\text { aldolase A }\end{array}$ & 23.57 & Cytoplasm & $\begin{array}{l}\text { Glycolysis and } \\
\text { gluconeogenesis }\end{array}$ \\
\hline 9 & P04406 & $\begin{array}{l}\text { Glyceraldehyde-3-phosphate } \\
\text { dehydrogenase }\end{array}$ & 4.72 & Cytoplasm & Glycolysis \\
\hline 31 & P30101 & $\begin{array}{l}\text { Protein disulfide-isomerase } \\
\text { A3 }\end{array}$ & 0.23 & Cytoplasm & $\begin{array}{l}\text { Cysteine-type } \\
\text { endopeptidase activity }\end{array}$ \\
\hline 33 & P14618 & $\begin{array}{l}\text { Pyruvate kinase isozymes } \\
\text { M1/M2 }\end{array}$ & 0.08 & Cytoplasm & Glycolysis \\
\hline 34 & Q9BWD1 & Acetyl-CoA acetyltransferase & 0.22 & Cytoplasm & $\begin{array}{l}\text { Acetyl-CoA } \\
\text { C-acetyltransferase activity }\end{array}$ \\
\hline 35 & P34949 & $\begin{array}{l}\text { Mannose-6-phosphate } \\
\text { isomerase }\end{array}$ & 0.73 & Cytoplasm & $\begin{array}{l}\text { Mannose-6-phosphate } \\
\text { isomerase activity }\end{array}$ \\
\hline 36 & P09972 & $\begin{array}{l}\text { Fructose-bisphosphate } \\
\text { aldolase C }\end{array}$ & 0.19 & Cytoplasm & $\begin{array}{l}\text { Glycolysis and } \\
\text { gluconeogenesis }\end{array}$ \\
\hline
\end{tabular}


Table II. Continued.

\begin{tabular}{|c|c|c|c|c|c|}
\hline $\begin{array}{l}\text { Spot } \\
\text { no. }\end{array}$ & $\begin{array}{l}\text { Accession } \\
\text { no. }\end{array}$ & $\begin{array}{l}\text { Protein } \\
\text { name }\end{array}$ & $\begin{array}{l}\text { Average } \\
\text { ratio }\end{array}$ & $\begin{array}{l}\text { Subcellular } \\
\text { location }\end{array}$ & $\begin{array}{l}\text { Main } \\
\text { function }\end{array}$ \\
\hline 38 & P50213 & $\begin{array}{l}\text { Isocitrate dehydrogenase } \\
\text { (NAD) subunit } \alpha, \\
\text { mitochondrial }\end{array}$ & 0.17 & Mitochondrion & $\begin{array}{l}\text { Tricarboxylic } \\
\text { acid cycle }\end{array}$ \\
\hline 39 & P37837 & Transaldolase & 0.43 & Cytoplasm & $\begin{array}{l}\text { Pentose-phosphate } \\
\text { pathway }\end{array}$ \\
\hline 40 & P07195 & $\begin{array}{l}\text { L-lactate dehydrogenase B } \\
\text { chain }\end{array}$ & 0.34 & Cytoplasm & $\begin{array}{l}\text { (S)-lactate + NAD+ } \\
=\text { pyruvate }+ \text { NADH. }\end{array}$ \\
\hline 43 & P11177 & $\begin{array}{l}\text { Pyruvate dehydrogenase E1 } \\
\text { component subunit } \beta \text {, } \\
\text { mitochondrial }\end{array}$ & 0.32 & Mitochondrion & $\begin{array}{l}\text { Pyruvate dehydrogenase } \\
\text { (acetyl-transferring) activity }\end{array}$ \\
\hline 44 & O00154 & $\begin{array}{l}\text { Cytosolic acyl coenzyme A } \\
\text { thioester hydrolase }\end{array}$ & 0.37 & Mitochondrion & $\begin{array}{l}\text { Fatty-acyl-CoA } \\
\text { binding }\end{array}$ \\
\hline 45 & P15121 & Aldose reductase & 0.31 & Cytoplasm & Catalytic efficiencies \\
\hline 46 & P00338 & $\begin{array}{l}\text { L-lactate dehydrogenase A } \\
\text { chain }\end{array}$ & 0.23 & Cytoplasm & $\begin{array}{l}\text { L-lactate dehydrogenase } \\
\text { activity }\end{array}$ \\
\hline 47 & P31937 & $\begin{array}{l}\text { 3-hydroxyisobutyrate } \\
\text { dehydrogenase, } \\
\text { mitochondrial }\end{array}$ & 0.33 & Mitochondrion & $\begin{array}{l}\text { 3-hydroxyisobutyrate } \\
\text { dehydrogenase activity }\end{array}$ \\
\hline 51 & P00491 & $\begin{array}{l}\text { Purine nucleoside } \\
\text { phosphorylase }\end{array}$ & 0.27 & Cytoplasm & Immune response \\
\hline 53 & P60174 & Triosephosphate isomerase & 0.47 & Cytoplasm & $\begin{array}{l}\text { Triose-phosphate } \\
\text { isomerase activity }\end{array}$ \\
\hline 55 & Q15185 & Prostaglandin E synthase 3 & 0.35 & Cytoplasm & Molecular chaperone \\
\hline 56 & Q99714 & $\begin{array}{l}\text { 3-hydroxyacyl-CoA } \\
\text { dehydrogenase type-2 }\end{array}$ & 0.41 & Mitochondrion & $\begin{array}{l}\text { 3-hydroxy-2-methylbutyryl-CoA } \\
\text { dehydrogenase activity }\end{array}$ \\
\hline 58 & P42126 & $\begin{array}{l}\text { Enoyl-CoA delta isomerase } \\
1, \text { mitochondrial }\end{array}$ & 0.38 & Mitochondrion & $\begin{array}{l}\text { Dodecenoyl-CoA } \\
\text { delta-isomerase activity }\end{array}$ \\
\hline 65 & Q01469 & $\begin{array}{l}\text { Fatty acid-binding protein, } \\
\text { epidermal }\end{array}$ & 0.27 & Cytoplasm & High specificity for fatty acids \\
\hline
\end{tabular}

Orlistat. It was downregulated more than 10-fold in SKOV3 cells treated with Orlistat compared to those without Orlistat, and MS/MS analysis revealed 15 matched peptides with $36 \%$ sequence coverage and a MOWSE score of 309 (Fig. 3).

Proteomic validation of identified proteins. The expression level of PKM2 was further validated by western blotting. Consistent with the observations in 2-DE analysis, PKM2 was downregulated in the Orlistat-treated SKOV3 cells compared with the parental SKOV3 cells. A similar change in the expression level of FASN was detected in SKOV3 cells treated with Orlistat (Fig. 5).

\section{Discussion}

Altered expression of lipid metabolic enzymes is a feature of various types of cancer, including those that develop in ovarian tissues (24). Highly proliferating cancer cells need to synthesize fatty acids de novo to continually provide lipids for membrane production. The synthesized fatty acids are also used for energy production through $\beta$-oxidation and lipid modification of proteins (Fig. 4). FASN, one of the key enzymes involved in de novo fatty-acid synthesis, was found to be overexpressed in various human types of cancer, including prostate, ovary, colon, and lung (25). FASN has been found to be essential for ovarian cancer cell survival and inhibition of FASN activity has been shown to have potential chemopreventive (26) and therapeutic applications (27).

In this study, we found that treatment with Orlistat, an inhibitor of FASN, promoted the apoptosis of SKOV3 cells (Fig. 1). We confirm the inhibitory effect of Orlistat on FASN by western blot analysis using the ovarian cancer cells (SKOV3) as a model, and we found that FASN was 2-fold downregulated after treatment with Orlistat. As shown in Fig. 5, we employed a 2-DE-based proteomics approach to annotate the altered proteins in the SKOV3 cells prior to and following treatment with Orlistat. Our proteomic analysis revealed a total of 71 differentially expressed proteins, which were associated with cell metabolism, proliferation and/or apoptosis.

Among them, Profilin 1, a member of the profilin family, also known as PFN1, was ubiquitous and upregulated more than 10-fold in SKOV3 cells after treatment with Orlistat. PFN1 was found to be involved in multiple cell behaviors, such as cell adhesion, growth, proliferation and signal transduction $(34,35)$. Moreover, 23 proteins were found differentially expressed related to metabolism. Among them, 


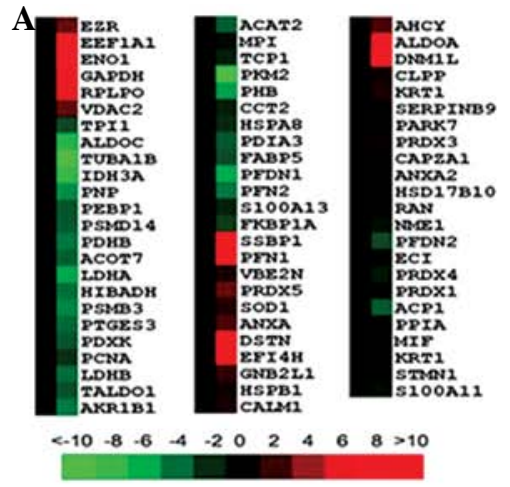

D

Matched peptides ghown in Bold red

1 YSKPHSEAGT AFIQTQQLHA NADTFLEHA CRLDIDSPPI TANTOITCT 51 TOPLSRSVET LEOCIKSOW VARLAFSHGT HEYHAETIKI VRTATESTAS 101 DPTLYRPVAV ALDTKGPETR TGLIKGSGTA EVELKKGATL KITLDKAME

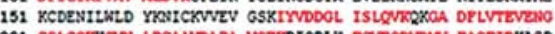

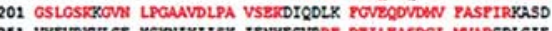

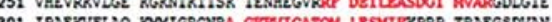

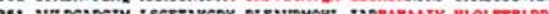

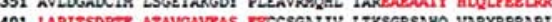
(5)

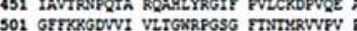

B
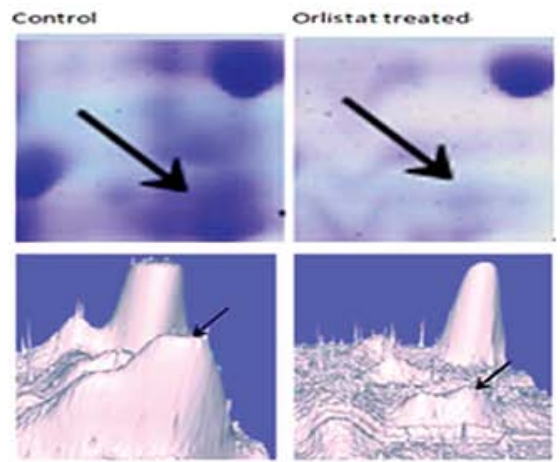

C

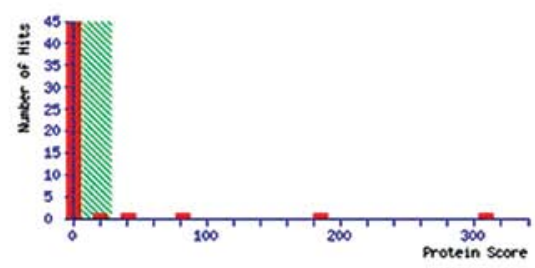

$\mathbf{E}$

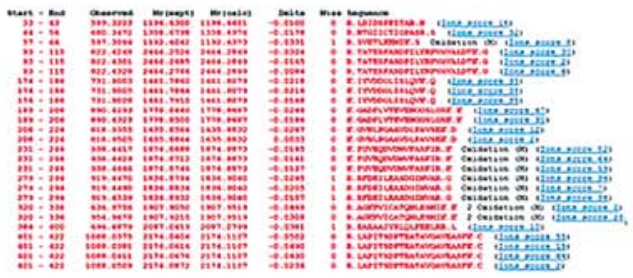

$\mathbf{F}$

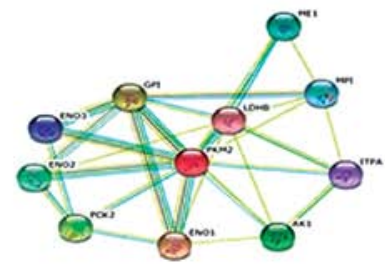

Figure 3. Identification and quantification of the proteins by LC-MS/MS Based SILAC method. (A) Clustering analysis of the identified proteins from SKOV3 cells treated with or without Orlistat. Protein cluster map generated by Cluster software. Expression of proteins in the normal group was constat at 0 , proteins upregulated in Orlistat-treated SKOV3 cells are in red, and the downregulated proteins are in green. The intensity of the color green or red corresponds to the degree of alteration, respectively, according to the color strip at the bottom of the figure. These data are derived from three independent analyses. (B) Cropped 2-DE gel images of PKM2. Downregulation by 12.25 -fold \pm 3.75 ( $t$ test, $\mathrm{p}<0.05$ ) was seen in Orlistat-treated SKOV3 cells. (C-E) Output of the LC MS/MS database using the MASCOT program. LC-MS/MS analysis revealed 15 matched peptides with 36\% sequence coverage and a MOWSE score of 309. The matched peptides are shown in bold red. (F) In silico protein interaction analysis. Regulated proteins involved in PKM2 regulation were analyzed for protein interactions using the web based software tool STRING.

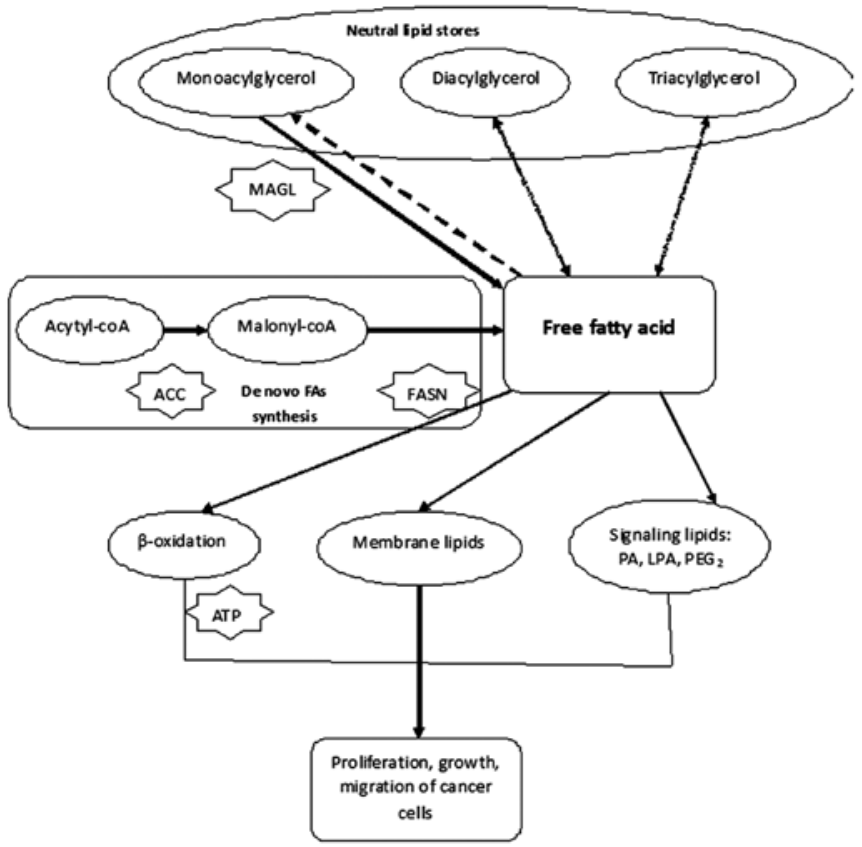

Figure 4. Free fatty acids can promote the proliferation, growth and migration of cancer cells. FASN plays a central role in regulation the synthesis of free fatty acids. So inhibition of FASN and the de novo fatty-acid synthesis pathways could be considered as novel strategies in antitumor territory ACC, acetyl-CoA carboxylase; FASN, fatty-acid synthase; MAGL, monoacylglycerol lipase; PA, phosphatidic acid; LPA, lysophosphatidic acid, and PGE2, prostaglandin E2, ATP, adenosine 5'-triphosphate.
A

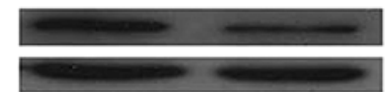

$\mathbf{B}$

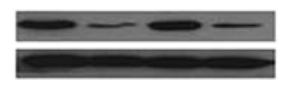

C

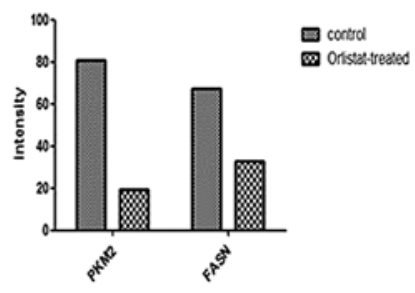

Figure 5. (A) Relative expression of FASN was further validated by western blot analysis. $\beta$-actin was used as a loading control. (B) Relative expression of PKM2 was further validated by western blot analysis. $\beta$-actin was used as a loading control. (C) Bands were analyzed by densitometry using Quality-One software (Bio-Rad). The $\mathrm{x}$-axis shows the average intensity from three individual experiments. Data are presented as the means $\pm \mathrm{SD}$.

pyruvate kinase (PK), a rate-limiting enzyme during glycolysis, catalyzes the production of pyruvate and adenosine 5 '-triphosphate (ATP) from phosphoenolpyruvate (PEP) and adenosine 5'-diphosphate (ADP) (28). Four mammalian PK isoenzymes (M1, M2, L and R) were found in normal adult cells. By contrast, PKM2 is found predominantly in the fetus as well as in tumor cells, where the abundance of other isoforms of PK is low. PKM2 can exist in either active tetramers or inactive dimers, but in tumor cells it predominantly occurs in dimers with low activity (29). Cancer cells universally express the M2 isoform of the glycolytic enzyme pyruvate kinase (PKM2), and previous studies have 
demonstrated that PKM2 expression is necessary for aerobic glycolysis and cell proliferation in vivo $(28,30)$. Knockdown of PKM2 using RNA interference significantly impairs cell growth in tissue culture, inhibition of PKM2 with peptide aptamers inhibits cell proliferation, and PKM2 expression is necessary for both aerobic glycolysis and tumor growth in vivo $(31,32)$. It has been proven that the downregulation of PKM2 activity in cancer cells aids in shunting key glycolytic intermediates toward pathways where they, in turn, are utilized as precursors for lipid, amino acid and nucleic acid synthesis. Therefore, the downregulation of PKM2 activity provides a purposeful divergence from catabolic metabolism aimed at energy production toward an anabolic state aimed at providing the needed resources for rapid cellular construction (33). Research has also shown that PKM2 plays a general role in caspase- and Bcl-independent apoptosis, thereby validating PKM2 as a promising, generally relevant target for the development of anticancer therapies with broad efficacy (34). In our study, PKM was downregulated more than 10-fold, confirming our hypothesis that Orlistat has antitumor abilities. Furthermore, significant downregulation of PKM2 after treatment with Orlistat was confirmed in the ovarian cancer cell line SKOV3 cells by western blot analysis.

In conclusion, using proteomic tools, we identified 71 differentially expressed proteins following Orlistat treatment of ovarian cancer cells. The functions of the differentially expressed proteins were correlated to apoptosis and/ or anti-proliferation cellular processes. These results support the hypothesis that Orlistat is a potential inhibitor of ovarian cancer and can be used as a novel assistant antitumor agent, combined with conventional surgical resection and chemotherapy.

\section{Acknowledgements}

This work was supported by the National Key Basic Research Program (973 Program) of China (2011CB910703).

\section{References}

1. Clemens MJ: Targets and mechanisms for the regulation of translation in malignant transformation. Oncogene 23: 3180-3188, 2004.

2. Bull JH, Ellison G and Patel A, et al: Identification of potential diagnostic markers of prostate cancer and prostatic intraepithelial neoplasia using cDNA microarray. Br J Cancer 84: 1512-1519, 2001.

3. Rashid A, Pizer ES, Moga M, et al: Elevated expression of fatty acid synthase and fatty acid synthetic activity in colorectal neoplasia. Am J Pathol 150: 201-208, 1997.

4. Gansler TS, Hardman W III, Hunt DA, et al: Increased expression of fatty acid synthase (OA-159) in ovarian neoplasms predicts shorter survival. Hum Pathol 18: 686-692, 1997.

5. GanslerTS,Hardman W III,HuntDA, et al:Immunohistochemical expression and prognostic significance of FAS and GLUT1 in bladder carcinoma. Anticancer Res 23: 335-339, 2003.

6. Nemoto T, Terashima S, Kogure M, et al: Overexpression of fatty acid synthase in oesophageal squamous cell dysplasia and carcinoma. Pathobiology 69: 297-303, 2001.

7. Kusakabe T, Nashimoto A, Honma K and Suzuki T: Fatty acid synthase is highly expressed in carcinoma, adenoma and in regenerative epithelium and intestinal metaplasia of the stomach. Histopathology 40: 71-79, 2002.

8. Piyathilake CJ, Frost AR, Manne U, et al: The expression of fatty acid synthase (FAS) is an early event in the development and progression of squamous cell carcinoma of the lung. Hum Pathol 31: 1068-1073, 2000.
9. Pizer ES, Lax SF, Kuhajda FP, Pasternack GR and Kurman RJ: Fatty acid synthase expression in endometrial carcinoma: correlation with cell proliferation and hormone receptors. Cancer 83: 528-537, 1998.

10. Alò PL, Visca P, Marci A, Mangoni A, Botti C and Di Tondo U: Expression of fatty acid synthase (FAS) as a predictor of recurrence in stage I breast carcinoma patients. Cancer 77: 474-482, 1996.

11. Pizer ES, Wood FD, Heine HS, Romantsev FE, Pasternack GR and Kuhajda FP: Inhibition of fatty acid synthase delays disease progression in a xenograft model of ovarian cancer. Cancer Res 56: 189-193, 1996.

12. Alò PL, Visca P, Botti C, et al: Immunohistochemical expression of human erythrocyte glucose transporter and fatty acid synthase in infiltrating breast carcinomas and adjacent typical/ atypical hyperplastic or normal breast tissue. Am J Clin Pathol 116: 129-134, 2001.

13. Alò PL, Visca P, Framarino ML, et al: Immunohistochemical study of fatty acid synthase in ovarian neoplasms. Oncol Rep 7: 1383-1388, 2000.

14. Costello LC and Franklin RB: 'Why do tumour cells glycolyse?': from glycolysis through citrate to lipogenesis. Mol Cell Biochem 280: 1-8, 2005

15. De Schrijver E, Brusselmans K, Heyns W, Verhoeven G and Swinnen JV: RNA interference-mediated silencing of the fatty acid synthase gene attenuates growth and induces morphological changes and apoptosis of LNCaP prostate cancer cells. Cancer Res 63: 3799-3804, 2003.

16. Menendez JA, Vellon L and Lupu R: Orlistat: From antiobesity drug to anticancer agent in Her-2/neu (erbB-2)-overexpressing gastrointestinal tumors? Exp Biol Med (Maywood) 230: 151-154, 2005.

17. Pemble CW IV, Johnson LC, Kridel SJ and Lowther WT: Crystal structure of the thioesterase domain of human fatty acid synthase inhibited by Orlistat. Nat Struct Mol Biol 14: 704-709, 2007.

18. Knowles LM, Axelrod F, Browne CD and Smith JW: A fatty acid synthase blockade induces tumor cell-cycle arrest by downregulating Skp2. J Biol Chem 279: 30540-30545, 2004.

19. Jemal A, Siegel R, Ward E, Hao Y, Xu J, Murray T and Thun MJ: Cancer statistics. CA Cancer J Clin 58: 71-96, 2008.

20. Raki M, Rein DT, Kanerva A and Hemminki A: Gene transfer approaches for gynecological diseases. Mol Ther 14: 154-163, 2006.

21. Liu R, Li Z, Bai S, et al: Mechanism of cancer cell adaptation to metabolic stress: proteomics identification of a novel thyroid hormone-mediated gastric carcinogenic signaling pathway. Mol Cell Proteomics 8: 70-85, 2009.

22. Tong A, Wu L, Lin Q, et al: Proteomics analysis of cellular protein alterations using a hepatitis B virus-producing cellular model. Proteomics 8: 2012-2023, 2008.

23. Liu R, Wang K, Yuan K, et al: Integrative oncoproteomics strategies for anticancer drug discovery. Expert Rev Proteomics 7: 411-429, 2009.

24. Medes G, Thomas A and Weinhouse S: Metabolism of neoplastic tissue. IV: A study of lipid synthesis in neoplastic tissue slices in vitro. Cancer Res 13: 27-29, 1953.

25. Witkowski A, Joshi AK and Smith S: Coupling of the de novo fatty acid biosynthesis and lipoylation pathways in mammalian mitochondria. J Biol Chem 282: 14178-14185, 2007.

26. Chirala SS and Wakil SJ: Structure and function of animal fatty acid synthase. Lipids 39: 1045-1053, 2004.

27. Kuhajda FP, Jenner K, Wood FD, Hennigar RA, Jacobs LB, Dick JD and Pasternack GR: Fatty acid synthesis: a potential selective target for antineoplastic therapy. Proc Natl Acad Sci USA 91: 6379-6383, 1994.

28. Christofk HR, Vander Heiden MG, Harris MH, et al: The M2 splice isoform of pyruvate kinase is important for cancer metabolism and tumour growth. Nature 452: 230-233, 2008.

29. Guminska M, Ignacak J, Kedryna T and Stachurska MB: Tumorspecific pyruvate kinase isoenzyme M2 involved in biochemical strategy of energy generation in neoplastic cells. Acta Biochim Pol 44: 711-724, 1997.

30. Mazurek S, Boschek CB, Hugo F and Eigenbrodt E: Pyruvate kinase type M2 and its role in tumor growth and spreading. Semin Cancer Biol 15: 300-308, 2005.

31. Dombrauckas JD, Santarsiero BD and Mesecar AD: Structural basis for tumor pyruvate kinase M2 allosteric regulation and catalysis. Biochemistry 44: 9417-9429, 2005. 
32. Spoden GA, Mazurek S, Morandell D, et al: Isotype-specific inhibitors of the glycolytic key regulator pyruvate kinase subtype M2 moderately decelerate tumor cell proliferation. Int J Cancer 123: 312-321, 2008.

33. Steták A, Veress R, Ovádi J, Csermely P, Kéri G and Ullrich A: Nuclear translocation of the tumor marker pyruvate kinase M2 induces programmed cell death. Cancer Res 67: 1602-1608, 2007.
34. Janke J, Schlüter K, Jandrig B, et al: Suppression of tumorigenicity in breast cancer cells by the microfilament protein profilin 1. J Exp Med 191: 1675-1686, 2000.

35. Wu N, Zhang W, Yang Y, et al: Profilin 1 obtained by proteomic analysis in all-trans retinoic acid-treated hepatocarcinoma cell lines is involved in inhibition of cell proliferation and migration. Proteomics 6: 6095-6106, 2006. 